\title{
Seed-Mediated Growth of Gold Nanorods: Limits of Length to Diameter Ratio Control
}

\author{
Christopher J. Ward, Robert Tronndorf, Alicia S. Eustes, \\ Maria L. Auad, and Edward W. Davis
}

Department of Polymer and Fiber Engineering, Auburn University, Auburn, AL 36849, USA

Correspondence should be addressed to Edward W. Davis; ewd0001@auburn.edu

Received 11 February 2014; Revised 16 March 2014; Accepted 16 March 2014; Published 14 April 2014

Academic Editor: William W. Yu

Copyright (c) 2014 Christopher J. Ward et al. This is an open access article distributed under the Creative Commons Attribution License, which permits unrestricted use, distribution, and reproduction in any medium, provided the original work is properly cited.

\begin{abstract}
The effects of the seed reaction conditions on the two-step seed-mediated growth of gold nanorods and the effect of gold and reducing agent content in the growth solution were evaluated. Results indicate that the reaction conditions used to produce the seeds have a significant impact on the aspect ratio of the gold nanorods produced. Increasing the concentration of gold or the reaction temperature in the seed production step results in lower length to diameter (aspect ratio) gold rods. In addition, the amount of prepared seed added to the growth solution impacts the rod aspect ratio, with increasing amounts of seed reducing the aspect ratio. The effects of reducing agent, ascorbic acid (AA), and gold content of the growth solution on the aspect ratio of the produced rods are strongly interrelated. There exists a minimum ascorbic acid to gold concentration below which rods will not form; however, increasing the ratio above this minimum results in shorter rods being formed. Characterization of nanorod growth is performed by UV-vis-NIR spectrophotometry and transmission electron microscopy (TEM).
\end{abstract}

\section{Introduction}

In metallic nanoparticles, the mean free path of electrons under standard conditions is generally about 10 to $100 \mathrm{~nm}$. As a result, metal nanoparticles display significantly different properties than either individual atoms or bulk materials [1]. One of the first applications for metallic nanoparticles was as a nanocatalyst [2]. Since then, other industries and processes potentially impacted by nanotechnology have been identified. These industries and processes include petroleum cracking, controlled release, mechanical enhancement, fireproofing, and control of gas permeation rates [3-8]. Gold nanoparticles, in particular, have been shown to be simple to functionalize and stable under oxidative environments, making them particularly attractive for biomedical applications such as biomedical sensing and cancer treatment and as carriers for bioactive compounds [9-13].

Gold nanoparticles are relatively easy to synthesize in the spherical form $[13,14]$; nanorods are significantly more difficult to produce. However, changing the shape of gold nanoparticles alters their interaction with resonant electromagnetic radiation, leading to potential advances in the field of sensors, contrast agents for optical detection, drug delivery, and cancer cell diagnostics [9]. In particular, the peak absorption can be varied across a wide range of frequencies by changing the length to diameter ratio. Both Kityk et al. and Ozga et al. have demonstrated nonlinear optical properties of Au doped thin films $[15,16]$. In these studies the surface plasmon resonance of the gold particles was shown to increase local charge transfer and determine the second order optical effects. In the last 20 years, several synthesis routes have been developed for the production of gold nanorods, including multiple template methods, variations of electrochemical methods, and photochemical methods [2, 12, 14]. However, these routes suffer from low yield of the nanorods, damage to the nanorods during production, and many times, low quality products due to a lack of substantial control over the growth. One of the simplest methods for production of gold nanorods 
in solution is the seed-mediated approach, where nanorods are grown from small spherical gold nanoparticles [12, 1719]. This approach utilizes a surfactant to stabilize growth and guide nanorod formation over the formation of other shapes. In addition, some variations of this technique utilize silver ions that promote the formation of nanorods but also reduce the aspect ratio $[20,21]$.

For many gold nanorod synthesis methods, tailoring the aspect ratio can be difficult. The strategy employed depends on the particular synthesis route, such as changes to the template used for template directed growth or changes in reaction time for photochemical or electrochemical growth $[2,12,14]$. With the seed-mediated approach, however, there are many more techniques for the tailoring of aspect ratios without reducing throughput, increasing reaction time, or limiting flexibility of the process. The seed-mediated process is fundamentally a wet chemical route and thus changes to reaction conditions including temperature and reactant concentrations should be facile routes to controlling product. For example, Smith et al. have demonstrated that trace contaminates such as iodide prevent nanorod formation [22]. Sao and Murphy demonstrated that increasing chloroauric acid amounts will result in higher aspect ratio nanorods until a critical level is reached where aspect ratio decreases again [23]. Increasing ascorbic acid amounts have been shown to decrease the aspect ratio of the nanorods, even leading to the production of alternate shapes at higher levels $[23,24]$. After becoming a pioneer of the seed-mediated growth of gold nanorods, Murphy et al. discovered that the introduction of silver nitrate improved yield but decreased aspect ratio [19-21]. However, the actual mechanism of the silver nitrate interaction is not yet completely understood. Pérez-Juste et al. have demonstrated that higher amounts of seed particles introduced into the growth solution will result in lower aspect ratio nanorods because of the introduction of more nucleation sites [25]. Finally, higher temperature of the growth solution has been documented to result in lower aspect ratio nanorods, due to decreased surfactant association tendency and resultant lower stabilization [26].

The goal of this work was to understand the limits of the process for producing nanorods of any aspect ratio. In the present work, we demonstrate the individual and interrelated effects of chloroauric acid $\left(\mathrm{HAuCl}_{4}\right)$ and ascorbic acid (AA). These factors control the rate of production of reduced gold in the reaction medium and are critical in controlling growth. Additionally, the seed production conditions were evaluated for their effects on the nanorod growth process.

Surface plasmon resonance occurs when the oscillation of the electromagnetic field, in a resonant frequency of light, forces conduction band electrons to begin to oscillate $[17,19]$. This results in two distinct absorption peaks in UV-vis-NIR spectra for colloidal gold nanorods. One peak corresponds with the transversal plasmon (along the shorter axis of the nanorods), while the other corresponds with the longitudinal plasmon (along the longer axis). The transversal plasmon peak is usually located around $520 \mathrm{~nm}$, but the longitudinal plasmon peak is red-shifted depending mainly on the aspect ratio of the nanorods; larger aspect ratios typically result in larger wavelength absorption peaks [27]. In this work UV-vis-NIR spectrophotometry was used to probe the samples and determine the presence and location of the longitudinal plasmon peak as a measure of rod formation and morphology.

\section{Experimental}

A general two-step procedure was used to prepare the nanorods. In the first step, called the seed reaction, seeds were formed by the reduction of chloroauric acid $\left(\mathrm{HAuCl}_{4}\right)$ by sodium borohydride $\left(\mathrm{NaBH}_{4}\right)$. In the second step, named growth, these seeds were added to a solution in which more $\mathrm{HAuCl}_{4}$ is reduced by L-ascorbic acid (AA). Surfactant, hexadecyltrimethylammonium bromide (CTAB), present in the growth step, served to direct growth of the seeds along one axis forming rods.

2.1. Preparation of Seed Solution. Seed solutions were prepared by slowly mixing $110,200,290$, or $380 \mu \mathrm{L}$ of chloroauric acid $\left(\mathrm{HAuCl}_{4}\right.$, Sigma-Aldrich, US, $8.6 \mu \mathrm{M}$ solution in DIwater) into $10 \mathrm{~mL}$ of CTAB (Sigma-Aldrich, US, $0.1 \mathrm{M}$ solution in DI-water). Upon inversion of the reaction vial three times by hand, this mixture turned a deep yellow color. To this mixture, $601 \mu \mathrm{L}$ of ice-cold sodium borohydride $\left(\mathrm{NaBH}_{4}\right.$, Fluka, $0.01 \mathrm{M}$ solution in DI-water) was then added and the mixture was swirled gently for two minutes to initiate the reduction of the gold. The solution immediately turned a murky brown color. The solution was then kept at a constant temperature $\left(28^{\circ} \mathrm{C}, 37^{\circ} \mathrm{C}\right.$, and $40^{\circ} \mathrm{C}$ were evaluated) for two hours and used.

2.2. Nanorod Growth Procedure. Growth solutions were prepared by adding $130 \mu \mathrm{L}$ of silver nitrate $\left(\mathrm{AgNO}_{3}\right.$, SigmaAldrich, $0.01 \mathrm{M}$ solution in DI water) to $10 \mathrm{~mL}$ of CTAB $(0.1 \mathrm{M}$ solution in DI-water) and slowly inverting the reaction vial by hand three times. To this mixture, 300, 440, 580, 720, or $840 \mu \mathrm{L}$ of $\mathrm{HAuCl}_{4}(0.0086 \mathrm{M}$ solution in DI-water $)$ was added and the mixture again inverted three times. The solution turned a deep yellow color within a minute of mixing. After subsequent addition of $25,40,55,70$, or $80 \mu \mathrm{L} \mathrm{L}$-ascorbic acid (AA, Sigma-Aldrich, 0.1 M in DI-water), the mixture was swirled by hand until it turned colorless. To this solution, $6,24,48,72,96,120,144,170$, or $194 \mu \mathrm{L}$ of the previously produced seed solution was added and the solution swirled again to promote dispersion. The solution was stored at $28^{\circ} \mathrm{C}$ for the duration of nanorod growth. The solution changed color over the course of the next two hours to a light pink.

It should be noted that stock solutions of chloroauric acid, $\mathrm{CTAB}$, and silver nitrate were produced and used over the course of the work but fresh solutions of sodium borohydride and ascorbic acid were produced for each synthesis. In addition, the CTAB solution required storage above room temperature to eliminate precipitation, and metallic instruments were not used with the chloroauric acid to prevent reduction of the gold. When a factor (temperature or reactant concentration) was evaluated, all other parameters were held constant. For the seed solution the standard conditions were $290 \mu \mathrm{L}$ of $8.6 \mu \mathrm{M} \mathrm{HAuCl}$ and $601 \mu \mathrm{L}$ of ice-cold $0.01 \mathrm{M}$ 


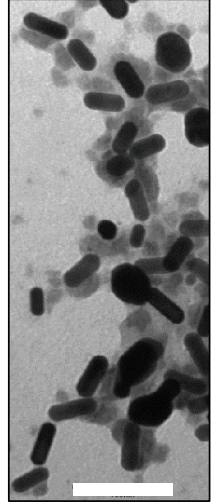

(a)

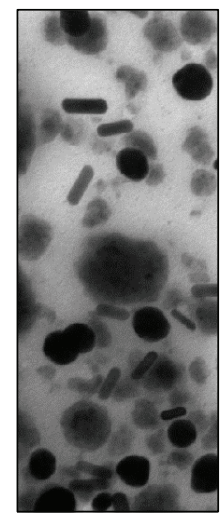

(b)

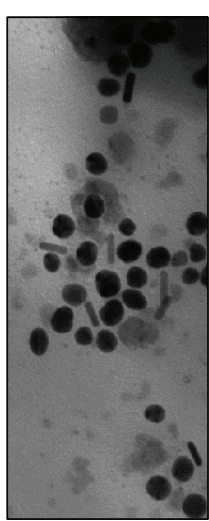

(c)

\begin{tabular}{lcc}
\hline Sample & Aspect ratio & $\begin{array}{c}\text { Peak absorption } \\
(\mathrm{nm})\end{array}$ \\
\hline $\mathrm{a}$ & 2.3 & 710 \\
$\mathrm{~b}$ & 3.4 & 804 \\
$\mathrm{c}$ & 3.8 & 831 \\
\hline
\end{tabular}

FIGURE 1: TEM images of gold nanorods of increasing aspect ratio, (a), (b), and (c). Table to right contains aspect ratio, from TEM samples, and location of peak absorption for each sample. The scale bar is $100 \mathrm{~nm}$.

$\mathrm{NaBH}_{4}$ in $10 \mathrm{~mL}$ of $0.1 \mathrm{M} \mathrm{CTAB}$ reacted at $37^{\circ} \mathrm{C}$ for two hours. For the growth solution the standard conditions were $130 \mu \mathrm{L}$ of $0.01 \mathrm{M} \mathrm{AgNO}, 580 \mu \mathrm{L}$ of $8.6 \mu \mathrm{M} \mathrm{HAuCl}_{4}, 55 \mu \mathrm{L}$ of $0.1 \mathrm{M}$ $\mathrm{AA}$, and $96 \mu \mathrm{L}$ of the previously prepared seed solution in $10 \mathrm{~mL}$ of $0.1 \mathrm{M} \mathrm{CTAB}$ reacted at $28^{\circ} \mathrm{C}$ for two hours.

2.3. Measurements and Characterization. Transmission electron microscopy (TEM) analysis was performed with a Zeiss EM 10C 10CR Transmission Electron Microscope. Samples were prepared for the TEM by centrifugation at 14,500 $\mathrm{g}$ for ten minutes, removal of supernatant, washing with DI water, and repeating. This final solution was placed on a carboncoated copper grid and the water was allowed to evaporate. After imaging, aspect ratios were determined by measuring the length and diameter of at least 30 rods randomly selected.

Each set of reaction conditions was replicated a minimum of 5 times. UV-vis-NIR spectra were obtained with a Shimadzu UV-2450 UV-vis spectrophotometer, resolution of $0.1 \mathrm{~nm}$, between absorption wavelengths of $1000 \mathrm{~nm}$ and $200 \mathrm{~nm}$, at intervals of $2 \mathrm{~nm}$. Peak absorbance wavelengths and absorbance at this wavelength were averaged over the replicates, and error bars shown in figures below are the standard error of the mean.

\section{Results and Discussion}

The wavelength of the longitudinal plasmon peak has been directly linked to the aspect ratio of the resultant gold nanorods $[28,29]$. In early analysis, we also found a positive correlation between the peak absorbance and the rod aspect ratio (Figure 1). Ni et al. have shown that while the peak absorption wavelength is linearly dependent on the aspect ratio the total absorption at that wavelength is a function of both rod concentration and rod diameter, with both smaller diameter rods and lower rod concentrations resulting in lower absorbance values [29]. However, reproducibility for the synthesis method studied here can be an issue. Thus the variation in the absorbance at the peak provides further confirmation that the results are reproducible. As UV-visNIR spectrophotometry is more accessible and quicker than TEM analysis, further analysis reported here will focus on the UV-vis-NIR measurements.

3.1. Effects of Seed and Seed Reaction Parameters. The amount of chloroauric acid present in the seed solution can significantly affect the final nanorod morphology. As shown in Figure 2(a), with increasing levels of gold ions present in the seed solution, both peak absorption wavelength and the absorbance at this wavelength generally decrease. With increased levels of gold ions, larger spheres were grown as seeds. These larger seeds yielded larger diameter nanorods of similar or shorter length than when using smaller seeds. This, in turn, led to smaller aspect ratios. These results mirror those of Gole and Murphy and Jiang and Pileni both of whom found that larger seeds result in lower aspect ratio rods [30, 31].

In addition to the effect of chloroauric acid concentration in the seed solution, the effect of the seed reaction temperature was evaluated. Three temperatures were evaluated: $28^{\circ} \mathrm{C}$, $37^{\circ} \mathrm{C}$, and $40^{\circ} \mathrm{C}$. While studies have examined the effects of the temperature used to grow the rods [32], no previous literature reports the effects of the seed solution reaction temperature on final rod morphology. To isolate the effect of the seed reaction temperature, all other parameters were held constant, including the reaction temperature used for rod growth, which was $28^{\circ} \mathrm{C}$. As seed growth temperature was increased, both peak absorption wavelength and the absorbance at this wavelength generally decreased, as shown in Figure 2(b). Literature suggests that when higher temperatures are used for the growth reaction, larger diameter rods are produced with lower aspect ratios due to the higher rate of gold reduction [32]. This increases the rate of gold growth limiting the ability to direct the growth in one dimension. Higher temperatures during seed growth similarly act to increase the concentration of reduced gold in the system leading to larger spheres. Larger seeds will grow into nanorods with larger diameters but shorter lengths. In addition, it has been suggested that, at higher temperatures, 


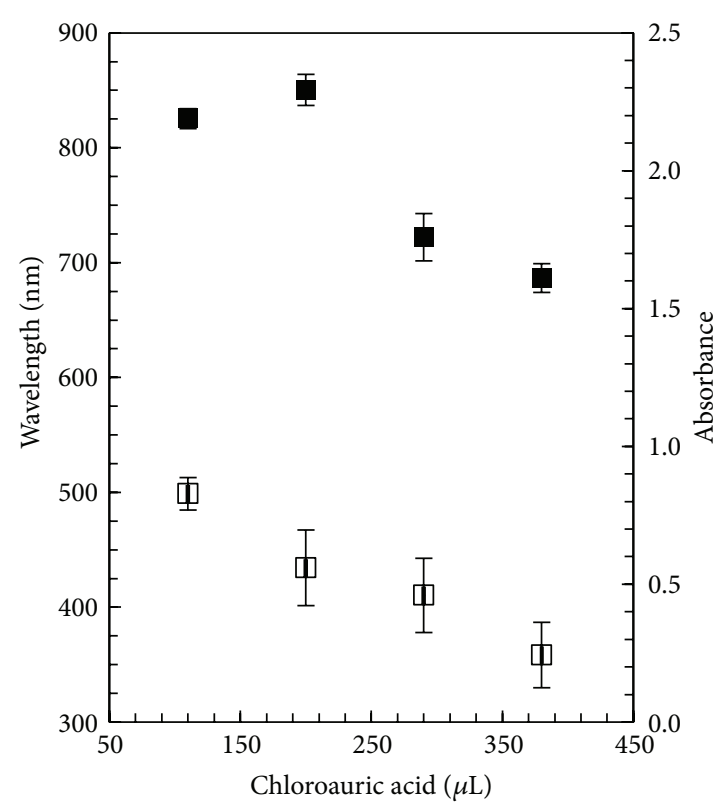

(a)

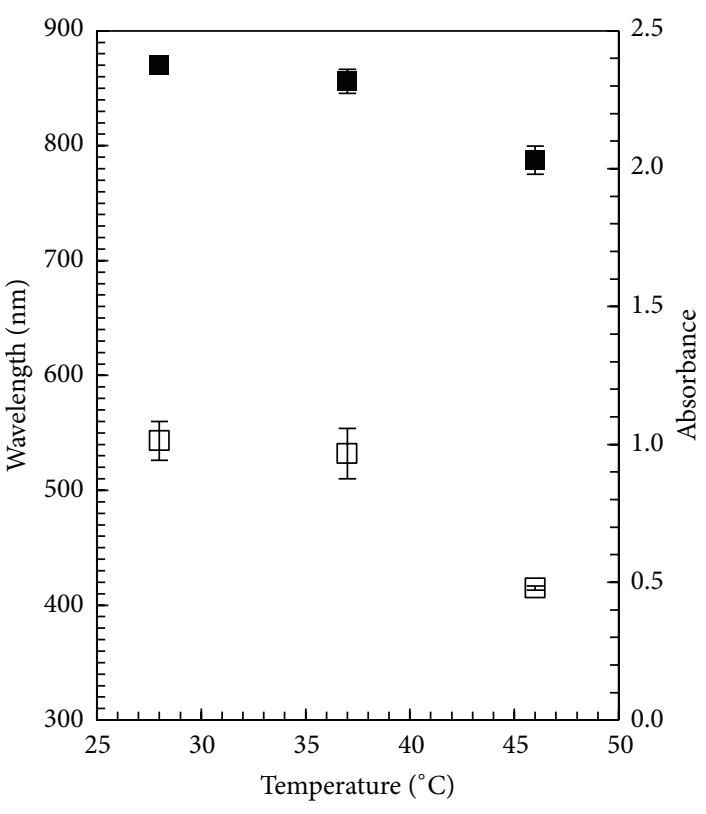

(b)

FIGURE 2: Effect of $\mathrm{HAuCl}_{4}$ content of seed solution (a) and seed reaction temperature (b) on the longitudinal plasmon resonance. Solid symbols are wavelength at peak absorbance, while open symbols are the absorbance of the growth solution at this wavelength. Error bars are standard error of the mean.

CTAB possesses an increased association tendency, forming more stable micelles but fewer of them [33]. Fewer seeds at a constant overall $\mathrm{Au}$ concentration result in larger seeds, and fewer nanorods are able to grow, reducing the yield.

To evaluate the idea that fewer seeds result in fewer nanorods, the effect of the amount of seed solution added to the rod growth solution was evaluated. If the amount of seed solution increases, there are more seeds present in the system for growth, resulting in more nanorods grown. However, as the vast majority of the gold in the system is added as $\mathrm{HAuCl}_{4}$ directly to the rod growth solution, the overall concentration of $\mathrm{Au}$ is only slightly affected by the amount of seed solution added. Thus, rod growth is limited, and shorter rods result; however, as the diameter remains constant, the aspect ratio drops. The results shown in Figure 3 support this hypothesis; increasing the seed concentration resulted in lower aspect ratio rods. The one exception to this trend was a system prepared with the lowest amount of seed solution. In this case the amount of seeds available for growth was not sufficient to allow directed growth and all facets of the seed grew. This resulted in large spherical particles, an effect previously reported by Li et al. [34].

3.2. Role of Rod Growth Conditions. The roles of the gold ion source $\mathrm{HAuCl}_{4}$ and the reducing agent, $\mathrm{AA}$, are intertwined. Ascorbic acid reduces the gold ions which then crystalize on the rod tips leading to growth. Too much reduced gold can overwhelm this process leading to other shapes, while too little reduced gold allows the effects of silver to dominate, also limiting the growth of the rods. While these two factors, $\mathrm{HAuCl}_{4}$ and $\mathrm{AA}$ concentration, are intertwined, they were

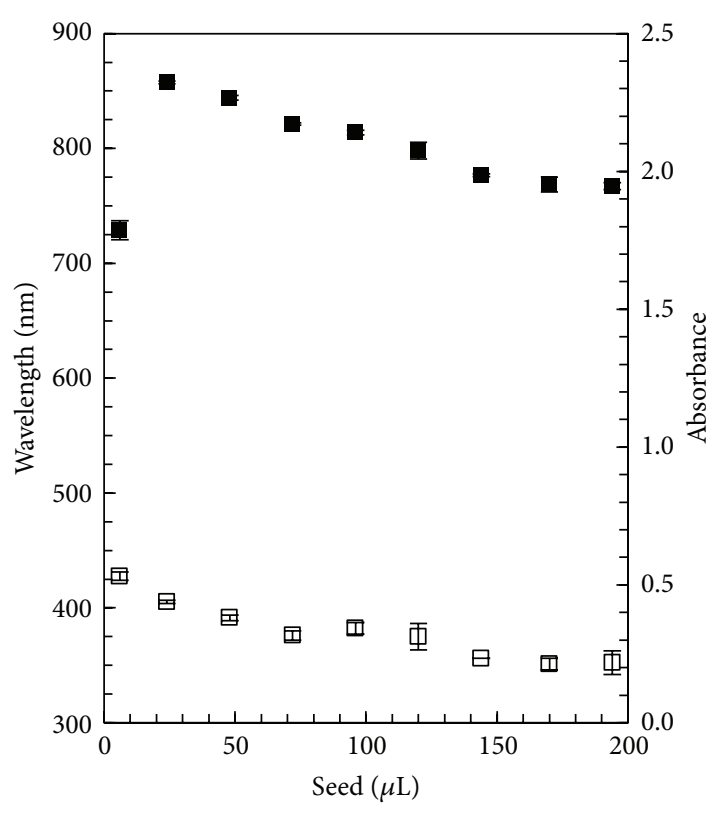

FIGURE 3: Peak absorbance of produced nanorods versus amount of seed added to growth solution. Solid symbols are wavelength at peak absorbance, while open symbols are the absorbance of the growth solution at this wavelength. Error bars are standard error of mean.

first evaluated in isolation. As Figure 4(a) demonstrates, as the amount of gold added to the rod growth solution was increased at first there was a rise in aspect ratio. However, continued increase in gold concentration actually resulted in no rod formation. This result is consistent with previous 


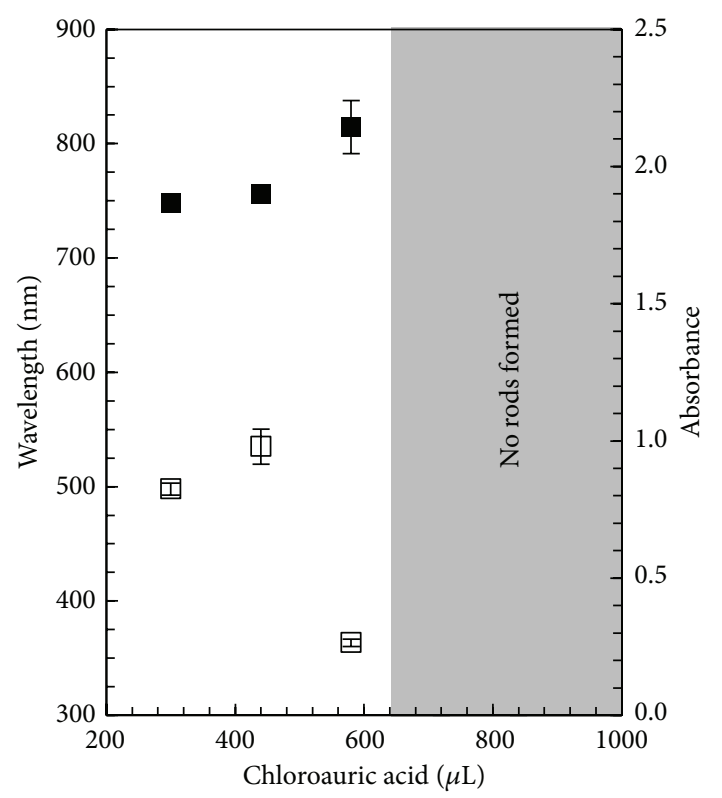

(a)

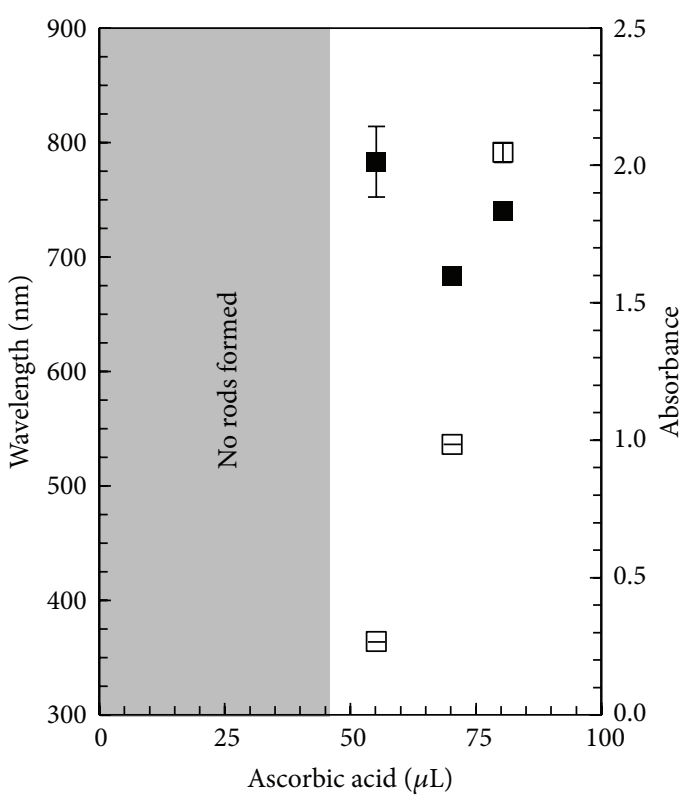

(b)

FIGURE 4: Effect of chloroauric acid and ascorbic acid content of growth solution on resulting nanorods. In (a), $\mathrm{HAuCl}_{4}$ is varied while holding AA content constant, while in (b), AA content is varied while holding $\mathrm{HAuCl}_{4}$ content constant. Solid symbols are wavelength at peak absorbance, while open symbols are the absorbance of the growth solution at this wavelength. Error bars are standard error of mean.

reports of a limiting $\mathrm{HAuCl}_{4}$ concentration [23]. During normal processing, the solution becomes colorless after the addition of AA, indicating reduction of the gold. When the highest amount of $\mathrm{HAuCl}_{4}, 840 \mu \mathrm{L}$, was used in our analysis, the solution still possessed a strong yellow color after addition of the AA, indicating an incomplete reduction of the gold ions. Interestingly, the solutions made with $720 \mu \mathrm{L}$ were of much more pale color and for one of the replicates colorless, suggesting that for these conditions $720 \mu \mathrm{L}$ is close to the critical value of $\mathrm{HAuCl}_{4}$. When full reduction did not occur, a peak around $400 \mathrm{~nm}$ was observed in the UV-vis spectra, samples with 720 and $840 \mu \mathrm{L}$ of $\mathrm{HAuCl}_{4}$. The presence of this peak and absence of a peak at higher wavelengths indicate that spheres but no rods were formed. Varying the amount of AA solution added from 25 to $80 \mu \mathrm{L}$ (Figure 4(b)) resulted in similar behavior. Below a critical concentration of AA, no rods were formed. While the requirement for a minimum amount of AA for the production of rods is clear, the trend at higher concentrations is not as clear. Gou and Murphy report an AA : Gold salt molar ratio of $1: 1$ [33], while here the ratio was varied from $1: 2$ to $2: 1$. Values less than $\sim 1: 1$ did not result in any rod formation.

Too much chloroauric acid results in similar growth data to when there is too little ascorbic acid. Interaction between the two levels was also examined. Nine different reaction conditions were evaluated, 560,580 , and $600 \mu \mathrm{L}$ of $\mathrm{HAuCl}_{4}$ pared with $53.3,55.2$, and $57.1 \mu \mathrm{L}$ of AA. These values were selected so that all reaction conditions are above the $1: 1$ level reported by Gou and Murphy [33]. Over the nine reaction conditions, the $\mathrm{AA}: \mathrm{HAuCl}_{4}$ ratio is varied from $1.033: 1$ to $1.186: 1$, a seemingly insignificant range. Results, shown in Figure 5 , indicate that as the amount of gold in the system was

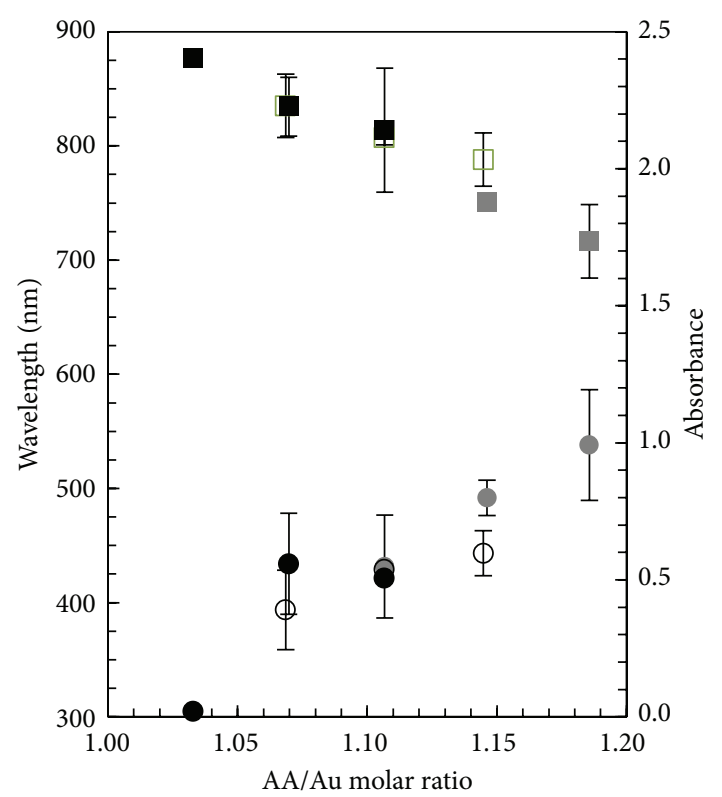

FIGURE 5: Effect of AA/Au molar ratio in growth solution on aspect ratio of resulting nanorods. Square symbols are wavelength at peak absorbance, while circles are the absorbance of the growth solution at the peak. Black symbols are for samples prepared using $600 \mu \mathrm{L}$ of $\mathrm{HAuCl}_{4}$ solution. Open symbols are for samples prepared using $580 \mu \mathrm{L} \mathrm{HAuCl}_{4}$. Grey symbols are for samples prepared using $560 \mu \mathrm{L}$ $\mathrm{HAuCl}_{4}$ solution. Error bars are standard error of mean.

increased, the aspect ratio increased. At a given level of gold, decreasing the AA concentration increased the rod aspect ratio. As gold and AA levels were increased simultaneously, 
keeping the ratio constant at $\sim 1.1067: 1$ the aspect ratio remained unchanged. This suggests that increasing the aspect ratio of the resulting rods can be achieved by increasing the gold concentration while decreasing the AA concentration. However, as there is a limit to the required $\mathrm{AA}: \mathrm{Au}$ ratio, below which no rods will form, there is a limit to the control of the aspect ratio by this method.

\section{Conclusion}

$\mathrm{HAuCl}_{4}$ concentration during the seed production has been shown to affect final rod morphology. Higher concentrations resulted in larger seeds and smaller aspect ratio rods. Temperature of seed growth also had a significant effect on rod formation; higher temperatures resulted in lower aspect ratio rods. A critical number of seeds must be added to the growth solution to promote the growth of rods. Too few seeds resulted in short or no rods. However, addition of additional seed, beyond the critical value, resulted in reduced aspect ratio rods. The roles of $\mathrm{HAuCl}_{4}$ and $\mathrm{AA}$ in the growth solution are strongly interrelated. Higher gold concentrations increased rod aspect ratio; however, there is a critical AA: $\mathrm{HAuCl}_{4}$ concentration ratio below which no rods will form. Dramatic effects were seen by relatively modest changes to relative amounts of $\mathrm{AA}$ and $\mathrm{Au}$ in the growth solution. Peak absorption wavelengths shifted from $\sim 875 \mathrm{~nm}$ to $\sim 715 \mathrm{~nm}$ as AA: Au ratios were increased from 1.03:1 to $1.18: 1$, an aspect ratio reduction of roughly $50 \%$.

\section{Conflict of Interests}

The authors declare that there is no conflict of interests regarding the publication of this paper.

\section{Acknowledgments}

The authors wish to recognize support from the Alabama Experimental Program to Stimulate Competitive Research (EPSCoR) and EPSCoR RID Seed Grants (Grant no. NNX07AL52A).

\section{References}

[1] A. P. Alivisatos, "Semiconductor clusters, nanocrystals, and quantum dots," Science, vol. 271, no. 5251, pp. 933-937, 1996.

[2] X. Huang, S. Neretina, and M. A. El-Sayed, "Gold nanorods: from synthesis and properties to biological and biomedical applications," Advanced Materials, vol. 21, no. 48, pp. 4880-4910, 2009.

[3] M. Du, B. Guo, and D. Jia, "Newly emerging applications of halloysite nanotubes: a review," Polymer International, vol. 59, no. 5, pp. 574-582, 2010.

[4] N. V. Patil, "Nanoclays make polymers stronger," Advanced Materials and Processes, vol. 163, no. 12, pp. 39-40, 2005.

[5] B. Xu, Q. Zheng, Y. Song, and Y. Shangguan, "Calculating barrier properties of polymer/clay nanocomposites: effects of clay layers," Polymer, vol. 47, no. 8, pp. 2904-2910, 2006.
[6] Y. M. Lvov, D. G. Shchukin, H. Möhwald, and R. R. Price, "Halloysite clay nanotubes for controlled release of protective agents," ACS Nano, vol. 2, no. 5, pp. 814-820, 2008.

[7] C. J. Ward, S. Song, and E. W. Davis, "Controlled release of tetracycline- $\mathrm{HCl}$ from halloysite-polymer composite films," Journal of Nanoscience and Nanotechnology, vol. 10, no. 10, pp. 6641-6649, 2010.

[8] H. Bönnemann and R. M. Richards, "Nanoscopic metal particles: synthetic methods and potential applications," European Journal of Inorganic Chemistry, vol. 2001, no. 10, pp. 2455-2480, 2001.

[9] X. Huang, I. H. El-Sayed, W. Qian, and M. A. El-Sayed, "Cancer cell imaging and photothermal therapy in the nearinfrared region by using gold nanorods," Journal of the American Chemical Society, vol. 128, no. 6, pp. 2115-2120, 2006.

[10] J. A. Li, D. Day, and M. Gu, "Ultra-low energy threshold for cancer photothermal therapy using transferrin-conjugated gold nanorods," Advanced Materials, vol. 20, no. 20, pp. 3866-3871, 2008.

[11] D. Pissuwan, T. Niidome, and M. B. Cortie, "The forthcoming applications of gold nanoparticles in drug and gene delivery systems," Journal of Controlled Release, vol. 149, no. 1, pp. 65-71, 2011.

[12] J. Pérez-Juste, I. Pastoriza-Santos, L. M. Liz-Marzán, and P. Mulvaney, "Gold nanorods: synthesis, characterization and applications," Coordination Chemistry Reviews, vol. 249, no. 1718, pp. 1870-1901, 2005.

[13] T. P. Ling, K. A. Razak, and A. A. Aziz, "Properties of gold nanoparticles synthesized in aqueous solution," AIP Conference Proceedings, vol. 1455, pp. 219-224, 2012.

[14] N. N. Long, L. van Vu, C. D. Kiem et al., "Synthesis and optical properties of colloidal gold nanoparticles," Journal of Physics: Conference Series, vol. 187, Article ID 012026, 2009.

[15] I. V. Kityk, J. Ebothé, I. Fuks-Janczarek et al., "Nonlinear optical properties of Au nanoparticles on indium-tim oxide substrate," Nanotechnology, vol. 16, article 1687, 2005.

[16] K. Ozga, T. Kawaharamura, A. Ali Umar et al., "Second order optical effects in Au nanoparticle-deposited $\mathrm{ZnO}$ nanocrystallite films," Nanotechnology, vol. 19, no. 18, Article ID 185709, 2008.

[17] A. V. Alekseeva, V. A. Bogatyrev, B. N. Khlebtsov, A. G. Mel'nikov, L. A. Dykman, and N. G. Khlebtsov, "Gold nanorods: synthesis and optical properties," Colloid Journal, vol. 68, no. 6, pp. 661-678, 2006.

[18] N. R. Jana, L. Gearheart, and C. J. Murphy, "Evidence for seedmediated nucleation in the chemical reduction of gold salts to gold nanoparticles," Chemistry of Materials, vol. 13, no. 7, pp. 2313-2322, 2001.

[19] C. J. Murphy, L. B. Thompson, D. J. Chernak et al., "Gold nanorod crystal growth: from seed-mediated synthesis to nanoscale sculpting," Current Opinion in Colloid and Interface Science, vol. 16, no. 2, pp. 128-134, 2011.

[20] B. Nikoobakht and M. A. El-Sayed, "Preparation and growth mechanism of gold nanorods (NRs) using seed-mediated growth method," Chemistry of Materials, vol. 15, no. 10, pp. 19571962, 2003.

[21] S. S. Seo, X. Wang, and D. Murray, "Direct monitoring of gold nanorod growth," Ionics, vol. 15, no. 1, pp. 67-71, 2009.

[22] D. K. Smith, N. R. Miller, and B. A. Korgel, "Iodide in CTAB prevents gold nanorod formation," Langmuir, vol. 25, no. 16, pp. 9518-9524, 2009. 
[23] T. K. Sau and C. J. Murphy, "Seeded high yield synthesis of short Au nanorods in aqueous solution," Langmuir, vol. 20, no. 15, pp. 6414-6420, 2004.

[24] S. K. Kang, I. Choi, J. Lee, Y. Kim, and J. Yi, "Investigation on shape variation of Au nanocrystals," Current Applied Physics, vol. 8, no. 6, pp. 810-813, 2008.

[25] J. Pérez-Juste, M. A. Correa-Duarte, and L. M. Liz-Marzán, "Silica gels with tailored, gold nanorod-driven optical functionalities," Applied Surface Science, vol. 226, no. 1-3, pp. 137-143, 2004.

[26] H. J. Park, C. S. Ah, W. Kim, I. S. Choi, K. Lee, and W. S. Yun, "Temperature-induced control of aspect ratio of gold nanorods," Journal of Vacuum Science and Technology A: Vacuum, Surfaces and Films, vol. 24, no. 4, Article ID 074604JVA, pp. 1323-1326, 2006.

[27] E. S. Kooij, W. Ahmed, C. Hellenthal, H. J. W. Zandvliet, and B. Poelsema, "From nanorods to nanostars: tuning the optical properties of gold nanoparticles," Colloids and Surfaces A: Physicochemical and Engineering Aspects, vol. 413, pp. 231238, 2012.

[28] T. A. El-Brolossy, T. Abdallah, M. B. Mohamed et al., "Shape and size dependence of the surface plasmon resonance of gold nanoparticles studied by Photoacoustic technique," European Physical Journal: Special Topics, vol. 153, no. 1, pp. 361-364, 2008.

[29] W. Ni, X. Kou, Z. Yang, and J. Wang, “Tailoring longitudinal surface plasmon wavelengths, scattering and absorption cross sections of gold nanorods," ACS Nano, vol. 2, no. 4, pp. 677-686, 2008.

[30] A. Gole and C. J. Murphy, "Seed-mediated synthesis of gold nanorods: role of the size and nature of the seed," Chemistry of Materials, vol. 16, no. 19, pp. 3633-3640, 2004.

[31] X. C. Jiang and M. P. Pileni, "Gold nanorods: influence of various parameters as seeds, solvent, surfactant on shape control," Colloids and Surfaces A: Physicochemical and Engineering Aspects, vol. 295, no. 1-3, pp. 228-232, 2007.

[32] R. Zou, Q. Zhang, Q. Zhao et al., "Thermal stability of gold nanorods in an aqueous solution," Colloids and Surfaces A: Physicochemical and Engineering Aspects, vol. 372, no. 1-3, pp. 177-181, 2010.

[33] L. Gou and C. J. Murphy, "Fine-tuning the shape of gold nanorods," Chemistry of Materials, vol. 17, no. 14, pp. 3668-3672, 2005.

[34] Q. Li, T. Bürgi, and H. Chen, "Preparation of gold nanorods of high quality and high aspect ratio," Journal Wuhan University of Technology, Materials Science Edition, vol. 25, no. 1, pp. 104-107, 2010. 

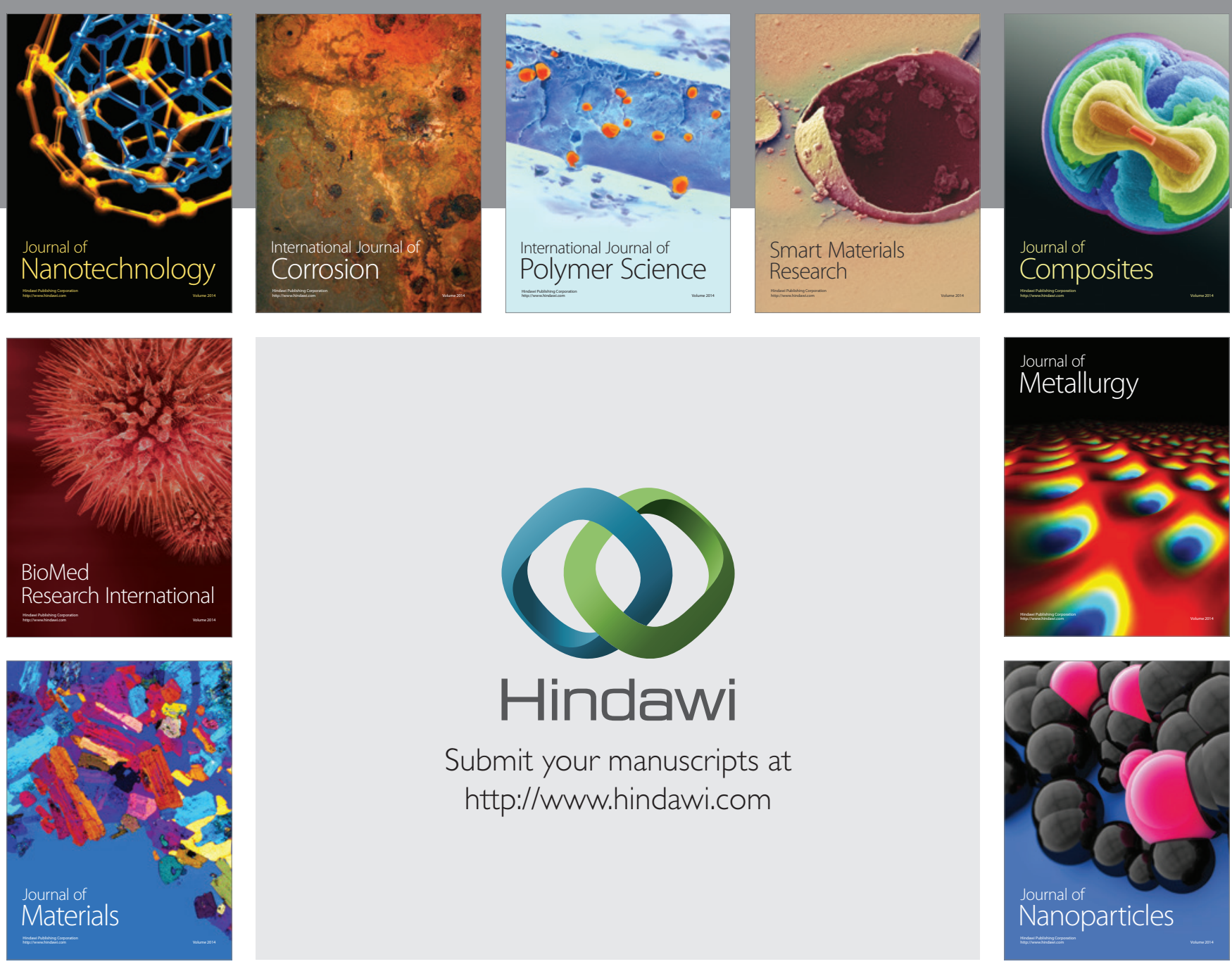

Submit your manuscripts at http://www.hindawi.com
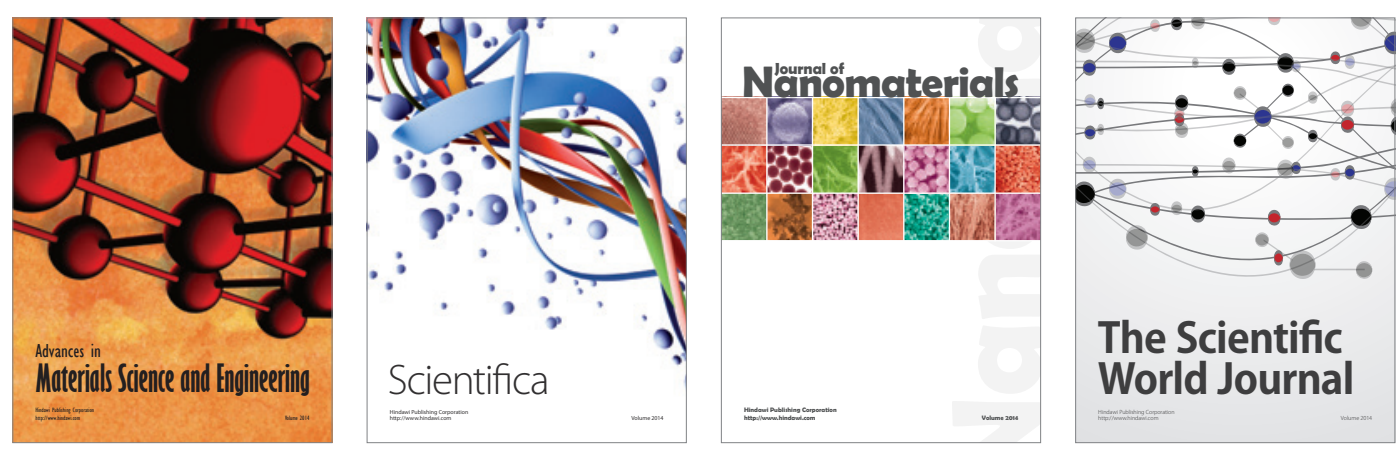

\section{The Scientific World Journal}
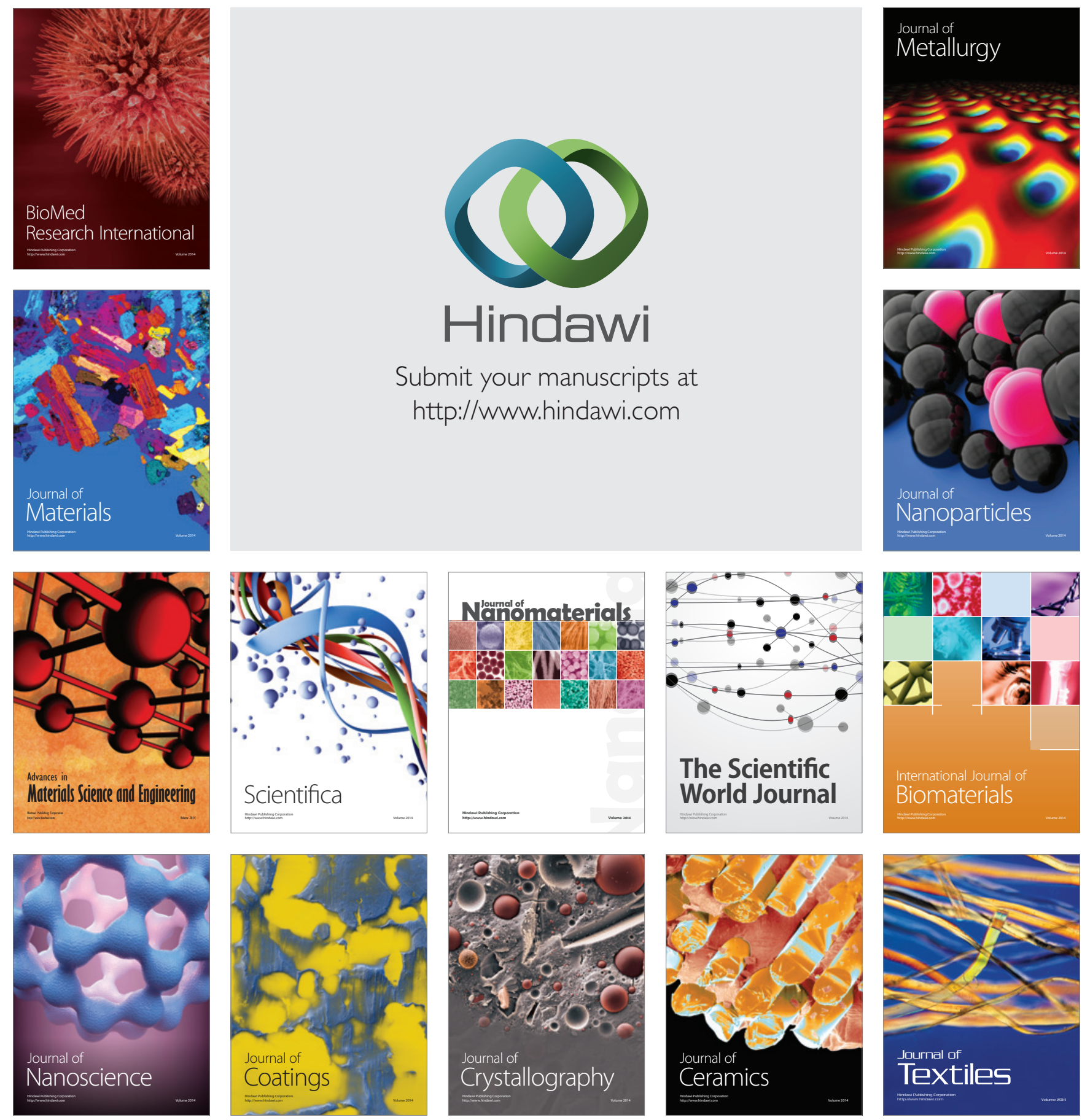